\title{
COVID-19 or not COVID-19? Compared characteristics of patients hospitalized for suspected COVID-19
}

\author{
Thomas Rogier $^{1}$ (D) Isabelle Eberl $^{1} \cdot$ Florian Moretto $^{1} \cdot$ Thibault Sixt $^{1} \cdot$ François-Xavier Catherine ${ }^{1}$. \\ Clémentine Estève $^{1}$ • Maroua Abdallahoui ${ }^{1}$ - Lucile Behague ${ }^{1}$. Antoine Coussement ${ }^{1} \cdot$ Lucas Mathey $^{1}$. \\ Sophie Mahy ${ }^{1}$ - Marielle Buisson ${ }^{1}$ - Arnaud Salmon-Rousseau ${ }^{1}$ • Michel Duong ${ }^{1}$ - Pascal Chavanet ${ }^{1}$. \\ Quentin Bernard $^{2}$ - Barbara Nicolas ${ }^{3}$ - Leila Benguella ${ }^{4}$ - Bernard Bonnotte ${ }^{3,5}$. Mathieu Blot ${ }^{1,6,7} \cdot$ Lionel Piroth $^{1,6,7}$
}

Received: 4 October 2020 / Accepted: 1 March 2021 / Published online: 8 March 2021

(C) The Author(s), under exclusive licence to Springer-Verlag GmbH Germany, part of Springer Nature 2021

\begin{abstract}
During an epidemic period, we compared patients hospitalized for initial suspicion of COVID-19 but for whom an alternative diagnosis was finally retained $(n=152)$ with those who had COVID-19 $(n=222)$. Most common diagnoses were another infectious disease and heart failure. COVID-19-negative patients were more often active smokers had less often cough, fever, and digestive symptoms, as compared to the 222 COVID-19-positive patients. They had higher median neutrophil and lymphocyte counts and lower CRP level. In multivariate analysis, no current smoking, neurocognitive disorder, myalgia, and fibrinogen $\geq 4 \mathrm{~g} /$ $\mathrm{L}$ were independently associated with a final diagnosis of COVID-19.
\end{abstract}

Keywords COVID-19, $\cdot$ SARS-CoV2, $\cdot$ Differential diagnosis, $\cdot$ Tobacco

\section{Introduction}

Since December 2019, the world is facing a pandemic situation, Coronavirus Disease 2019 (COVID-19), a manifestation of infection with Severe Acute Respiratory Syndrome Coronavirus2 (SARS-CoV-2) [1]. COVID-19 clinical manifestations are mainly respiratory. Almost $5 \%$ of patients develop severe pneumonia with acute respiratory distress syndrome (ARDS), with a mortality rate as high as $50 \%$ [2-4].

In the literature, there are many studies on patients suffering from COVID-19. By contrast, we lack data on the patients who were initially suspected of suffering from COVID-19, but for whom the diagnosis was finally ruled out. This is of

Thomas Rogier and Isabelle Eberl contributed equally to this work.

Thomas Rogier

thomas.rogier@chu-dijon.fr

1 Infectious Diseases Department, Dijon Bourgogne University Hospital, 14 rue Paul Gaffarel, 21079 Dijon, France

2 Department of Clinical Nephrology, Dijon Bourgogne University Hospital, Dijon, France

3 Department of Internal Medicine and Clinical Immunology, Dijon Bourgogne University Hospital, Dijon, France particular interest at the collective level since hospitalization flows and wards need to be reorganized because of the epidemic, and to avoid overloading the system by suspicions which will finally turn out wrong. It is also of particular interest at the individual level, since the isolation to avoid contamination and the potential delay in clinical biological and radiological diagnostic procedures may be associated with lost opportunities for patients suffering from diseases other than COVID-19.

Thus, the aim of this study was to describe and compare the population of patients hospitalized for a suspicion of COVID19 in an epidemic context in the University Hospital of Dijon, France.

4 Department of Rheumatology, Dijon Bourgogne University Hospital, Dijon, France

5 Univ. Bourgogne Franche-Comté, INSERM, EFS BFC, UMR1098, RIGHT Interactions Greffon-Hôte-Tumeur/Ingénierie Cellulaire et Génique, F-21000 Dijon, France

6 Univ. Bourgogne Franche-Comté, LNC UMR1231, F-21000 Dijon, France

7 INSERM, LNC UMR 1231, FCS Bourgogne-Franche Comté, LipSTIC LabEx, F-21000 Dijon, France 


\section{Patients and methods}

\section{Study design and patient selection}

Patients hospitalized with suspected COVID-19 in the COVID19 diagnostic unit and the Infectious Diseases (ID) department of the University Hospital of Dijon between February 23, 2020, and April 28, 2020, were retrospectively included. This period corresponded to the first wave of COVID-19 in Burgundy (France), a massive SARS-CoV-2 outbreak.

Patients were considered as COVID-19 positive if at least one RT-PCR assay was positive on a respiratory sample during the hospitalization. Patients were considered as COVID19-negative if all RT-PCRs assays were negative, without any other strong argument for COVID-19 at the end of the hospitalization. Patients with negative real-time reverse-transcriptase-polymerase-chain-reaction (RT-PCR) assays but with suggestive chest $\mathrm{CT}$ and without an alternate diagnosis were considered indeterminate and were not included. Patients with negative RT-PCRs assay but with suggestive chest computed tomography (CT) and alternate diagnosis were considered as COVID-19 negative. At the beginning of the epidemics, all patients suspected of COVID-19 were hospitalized in the ID department. Then, from the 18th of March, such patients were first admitted in a COVID-19 unit of diagnosis, whose functions were to sort out patients based on the results of RT-PCR and chest-CT. From that date on, the ID department only received patients with confirmed COVID-19. Patients initially admitted in intensive care unit were not included.

\section{Variables of interest and data collection}

Demographic data were collected, as well as the major comorbidities. Clinical data reviewed were the worst characteristics during the first $24 \mathrm{~h}$ of the stay. Biological parameters were determined from the first blood sampling during the first 48 hours of the hospitalization. The RT-PCR assay was performed on nasopharyngeal secretions and/or another respiratory sample. Chest CT findings were classified into evocative or not of COVID-19, according to the radiologist's interpretation. The severity of the impairment was classified according to the recommendations of the French Society of Radiology as: slight (including minimal), moderate or severe (including critical) $[5,6]$.

\section{Statistical analysis}

Continuous variables were expressed as means +/- SDs or medians and inter-quartile ranges (IQRs). Categorical variables were described by frequencies and percentages. Univariate analysis consisted of comparisons between variables, according to COVID-19 status, performed using the chi-square test (or Fisher's exact test) for percentages, Student's test for means and Wilcoxon Mann-Whitney test for medians and IQRs. The discriminant thresholds of the quantitative variables were determined using ROC curves. The statistically significant variables with $p<0.2$ in univariate analysis were then used in a multivariate step-down model to determine a predictive model for the diagnosis of COVID-19. To limit classification bias, a sensitivity analysis was performed by classifying patients with moderate or severe impairment on chest CT as COVID-19 positive. Statistical significance was defined as a $p$-value $<0.05$. Statistical analyses were performed with Epi Info 7.2 and XLSTAT software 2020.

\section{Results}

As depicted in the flowchart (Fig. 1), among the 304 patients hospitalized in the ID department and the 379 patients

Fig. 1 Flowchart

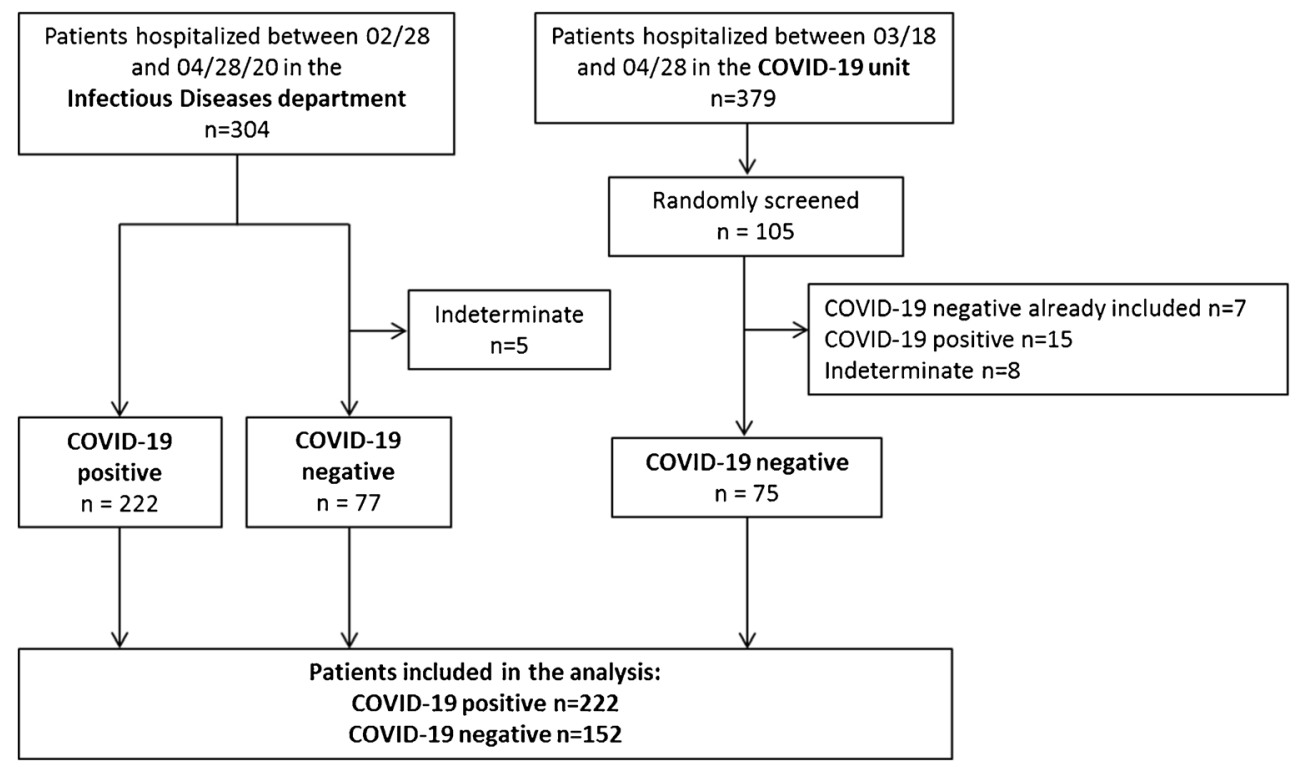


hospitalized in the COVID-19 diagnostic unit, 374 patients (152 COVID-19 negative and 222 COVID-19 patients) were included and compared.

Characteristics of COVID-19-negative patients were depicted in Table 1. More than $75 \%$ were over 50 years old. Seventy-eight (51.3\%) had fever and dyspnea. Chest CT was not suggestive of COVID-19 in more than $75 \%$ of patients. Main final diagnoses were another infectious disease for 68 $(44.7 \%)$ patients (pneumonia for $31(20.4 \%)$ patients, bacteremia for $12(7.9 \%)$ patients), heart failure for $13(8.6 \%)$ patients, and exacerbation of a chronic pulmonary disease for 12 (7.9\%) patients (Table 2).

In multivariate analysis, four variables were independently associated with a final diagnosis of COVID-19: neurocognitive disorder, no current smoking, myalgia, and a fibrinogen level greater than $4 \mathrm{~g} / \mathrm{L}$.

Among COVID-19-negative patients, two had a delay in surgical management (one spinal cord hematoma and one appendicular peritonitis). In addition, 5 patients with bacteremia had not been given antibiotics on admission to hospital. Finally, a late diagnosis of pneumocystis pneumonia leading to HIV infection at the AIDS stage was made in a patient with an interstitial syndrome and several negative RT-PCRs.

\section{Discussion}

Herein we present what is, to our knowledge, the first study of patients hospitalized with a suspicion of COVID-19 but a final alternative diagnosis. Among the 404 patients hospitalized with an initial suspicion of COVID-19, 154 (nearly 40\%) were negative and considered with an alternative diagnosis.

First, we showed that about $40 \%(n=68)$ of patients initially suspected as COVID-19 patients, in fact had another infectious disease, especially a bacteremia for $12(7.9 \%)$ patients and a urinary tract infection for 7 (4.6\%) patients, highlighting the importance of widespread blood cultures and cytobacteriological testing of urine. Furthermore, almost one tenth of the patients had acute heart failure and more than $50 \%$ had NT-pro-BNP $>1000 \mathrm{pg} / \mathrm{mL}$, highlighting that heart failure is therefore a differential diagnosis of COVID-19 infection, especially in elderly patients, even though heart failure is also a common condition associated with higher risk of in-hospital mortality among COVID-19 hospitalized patients [7-9].

In the context of a pandemic, it seems important to have simple ways to sort COVID-19-infected patients from uninfected ones. This could allow us to avoid overloading the health care system and avoid significant loss of opportunity in uninfected patients. Accordingly, 8 patients out of the 152 COVID-19-negative had a delay in management even though no death was reported due to this misdiagnosis. COVID-19-negative patients appear to have been symptomatic for a shorter period of time before being hospitalized, and to have less fever, digestive symptoms and myalgia, which has been reported previously [10]. Also, it is interesting to point out that COVID-19-negative patients had a higher neutrophil and lymphocyte count, which has also been mentioned $[10,11]$.

Up to now, screening patients with suspected COVID-19 have relied on nasopharyngeal RT-PCR and chest CT. When the results of RT-PCR and chest CT are combined, a sensitivity of $88 \%$ and a specificity of $100 \%$ may be achieved [11]. However, chest CT is a radiation procedure and its use for screening purposes during a pandemic may result in a longer time frame for other patients. Other means of screening need to be evaluated. In our multivariate analysis, we showed that in the context of pandemic, not smoking, neurocognitive disorder, having a fibrinogen $\geq$ $4 \mathrm{~g} / \mathrm{L}$, and/or myalgia largely increased the probability of COVID-19.

In our study, whereas the prevalence of active smokers in hospitalized COVID-19-negative patients was close to that reported in the whole population in France (24.0\%) [12], there were nearly 5-times fewer active smokers in patients hospitalized with COVID-19. Whether active smoking could prevent COVID-19 is still debated [13]. On the one hand, cigarette smoking seems to induce up-regulation of the natural SARS-CoV-2 receptor angiotensin-converting enzyme 2 (ACE2) in human cells $[14,15]$. On the other hand, ACE2 allows the conversion of angiotensin II into angiotensin 1-7 (Ang1-7), a peptide with antiinflammatory properties $[16,17]$. Thus, smokers could have at a greater risk of infection but at lower risk of developing a severe form of the disease $[18,19]$.

Our study has several limitations. First, our findings are themselves the reflection of specific epidemiological and organizational conditions. The suspicion of COVID-19 was not standardized, but was dependent on physician in charge of patients, who referred the patients to the hospital. In addition, the study was conducted when the epidemic peaked in France, and results would be different with circulation of other respiratory viruses (e.g., influenza, respiratory syncytial virus) responsible for flu syndrome. Moreover, 21 (13.8\%) COVID19-negative patients had no radiological examination; chest CT was not performed in $35.5 \%$ and $22(22.4 \%)$ had evocative impairment on the chest CT. Nevertheless, sensitivity analysis confirmed our results. In addition, the higher frequency of cognitive disorders in COVID-19 patients might be explained by the admission of older patients from nursing homes. However, we did not find any association with these two variables, possibly by a lack of power of the study. Patients with neurocognitive disorders also have less ability to comply with preventive measures, which could explain their propensity to get COVID-19. This association may also reflect a frailty which leads to an increased risk of COVID-19 
Table 1 Comparison between patients with (COVID-19 +) and without (COVID-19 -) COVID-19

\begin{tabular}{|c|c|c|c|}
\hline Characteristics & COVID-19+ & COVID-19 - & $p$ \\
\hline Number & 222 & 152 & NA \\
\hline \multicolumn{4}{|l|}{ Demographic data } \\
\hline $\operatorname{Sex} \mathrm{F}^{*}(n, \%)^{* *}$ & $99(44.6)$ & $82(53.4)$ & 0.09 \\
\hline Age at admission (mean, sd) & $70.2(17.0)$ & $67.8(20.0)$ & 0.46 \\
\hline Current smoking status $(n, \%)^{* *}$ & $8(4.4)$ & $28(27.2)$ & $<0.001$ \\
\hline Cardiovascular disease $(n, \%)^{* *}$ & $133(59.9)$ & $75(49.3)$ & 0.04 \\
\hline Obesity $(n, \%)$ & $34(16.0)$ & $17(11.2)$ & 0.22 \\
\hline Cirrhosis $(n, \%)$ & $1(0.5)$ & $8(5.3)$ & 0.003 \\
\hline Chronic kidney disease $(n, \%)$ & $18(8.1)$ & $12(7.9)$ & 1 \\
\hline Immunodeficiency $(n, \%)$ & $19(8.6)$ & $9(5.9)$ & 0.43 \\
\hline Chronic pulmonary disease $(n, \%)^{* *}$ & $30(13.5)$ & $32(21.1)$ & 0.05 \\
\hline Neurocognitive disorders $(n, \%)^{* *}$ & 48 (21.6) & $13(8.6)$ & $<0.001$ \\
\hline \multicolumn{4}{|l|}{ Clinical and paraclinical data } \\
\hline \multicolumn{4}{|l|}{ Clinical } \\
\hline Number of days between admission and symptoms onset (median, IQR)** & $6(3-9)$ & $3(0-7)$ & $<0.001$ \\
\hline Myalgia $(n, \%)^{* *}$ & $82(36.9)$ & $21(14.0)$ & $<0.001$ \\
\hline Confusion $(n, \%)$ & $37(16.7)$ & $18(11.8)$ & 0.23 \\
\hline $\operatorname{Cough}(n, \%)^{* *}$ & $146(65.8)$ & $76(50.0)$ & 0.003 \\
\hline Fever $(n, \%)^{* *}$ & $169(76.1)$ & $78(51.3)$ & $<0.001$ \\
\hline Dyspnea $(n, \%) * *$ & $137(61.7)$ & $78(51.3)$ & 0.06 \\
\hline Digestive symptoms (diarrhea and/or vomiting) $(n, \%)^{* *}$ & $66(29.7)$ & $18(11.8)$ & $<0.001$ \\
\hline NEWS2* at admission (median, IQR) & $5(3-8)$ & $5(3-8)$ & 0.87 \\
\hline qSOFA* at admission (median, IQR)** & $0(0-1)$ & $1(0-1)$ & 0.049 \\
\hline CRB65* at admission (median, IQR) & $1(1-1)$ & $1(0-2)$ & 0.47 \\
\hline RT-PCR (mean, sd) & MD & $1.3(0.7)$ & \\
\hline \multicolumn{4}{|l|}{ Site $(n, \%)$} \\
\hline - Nasopharyngeal & MD & $134(88.2)$ & NA \\
\hline - Depth & MD & $4(2.6)$ & NA \\
\hline$-\geq 2$ sites & MD & $14(9.2)$ & NA \\
\hline \multicolumn{4}{|l|}{ Biological (median, IQR) } \\
\hline Neutrophil $(\mathrm{G} / \mathrm{L})^{* *}$ & $4.7(3.3-6.6)$ & $7.6(5.1-10.5)$ & $<0.001$ \\
\hline Lymphocyte $(\mathrm{G} / \mathrm{L}) * *$ & $0.9(0.6-1.4)$ & $1.1(0.7-1.7)$ & 0.02 \\
\hline Fibrinogen $(\mathrm{g} / \mathrm{L})^{* *}$ & $6.0(5.3-6.8)$ & $5.3(4-6.5)$ & $<0.001$ \\
\hline Urea $(\mathrm{mmol} / \mathrm{L})$ & $6.6(4.8-10.4)$ & $7(4.8-11.4)$ & 0.30 \\
\hline Creatinine $(\mu \mathrm{mol} / \mathrm{L})$ & $76(60-100)$ & $77(61-100)$ & 0.45 \\
\hline $\mathrm{CRP} *(\mathrm{mg} / \mathrm{L})^{* *}$ & $83.7(37.7-127)$ & $57.3(13-126)$ & 0.008 \\
\hline $\mathrm{ALAT}^{*} \geq 3 \mathrm{~N}(n, \%)$ & $12(7.1)$ & $10(8.3)$ & 0.82 \\
\hline \multicolumn{4}{|l|}{ Radiological data $(n, \%)$} \\
\hline No radiological examinations performed & MD & $21(13.8)$ & NA \\
\hline Chest radiograph & MD & $39(27.7)$ & NA \\
\hline - Non-evocative & MD & $25(64.1)$ & NA \\
\hline - Lobar pneumonia & MD & $7(17.9)$ & NA \\
\hline - Potentially compatible with COVID-19 & MD & $7(17.9)$ & NA \\
\hline Chest $\mathrm{CT}^{*}$ & $164(73.9)$ & $98(64.5)$ & 0.07 \\
\hline - Non evocative & $17(10.4)$ & $76(77.6)$ & $<0.001$ \\
\hline \multicolumn{4}{|l|}{ - Evocative } \\
\hline - Slight & $19(11.6)$ & $10(10.2)$ & 0.84 \\
\hline - Moderate & $53(32.3)$ & $10(10.2)$ & $<0.001$ \\
\hline - Severe & $75(45.7)$ & $2(2.0)$ & $<0.001$ \\
\hline \multicolumn{4}{|l|}{ Evolution } \\
\hline Length of hospital stay (median, IQR) & $6(3-9.5)$ & $4(2-10)$ & 0.06 \\
\hline In-hospital mortality or transfer to intensive care unit $(n, \%)$ & $64(28.8)$ & $14(9.2)$ & $<0.001$ \\
\hline
\end{tabular}

* F female; NEWS National Early Warning Score; $q S O F A$ quick sepsis-related organ failure assessment; $C R B$ confusion respiratory blood; $C R P$ Creactive-protein; $A L A T$ alanine aminotransferase; $C T$ computed tomography; $M D$ missing data

**Variables included in multivariate model

requiring hospitalization. Thus, 13 patients (3\% of the included population) were excluded due to an indeterminate status (negative PCR but chest CT-scan suggestive of COVID-19). Accordingly, this may lead to a bias that could have been avoided if serology or multiplex PCR had been available.
Finally, anosmia and loss of taste were not collected though they were described as highly predictive of the presence of the virus [20]. Indeed, these data were not often collected from the sickest patients; many other patients were unable to describe these symptoms because of their age or altered brain function. 
Table 2 Final diagnosis for COVID-19-negative patients

\begin{tabular}{ll}
\hline Infectious disease $(n, \%)$ & 68 \\
& $(44.7)$ \\
\hline - Pneumonia & 31 \\
& $(20.4)$ \\
- Bacteremia & $12(7.9)$ \\
- Urinary tract infection & $7(4.6)$ \\
- Upper respiratory tract viral infection & $7(4.6)$ \\
- Digestive viral infection & $3(2.0)$ \\
- Diverticulitis & $3(2.0)$ \\
- Other & $5(3.2)$ \\
Cardiovascular or respiratory disease $(n, \%)$ & 33 \\
& $(21.7)$ \\
- Heart failure & $13(8.6)$ \\
- Exacerbation of chronic pulmonary disease & $12(7.9)$ \\
- Other & $5(3.2)$ \\
- Pulmonary embolism & $3(2.0)$ \\
Unlabelled diagnosis $(n, \%)$ & 18 \\
& $(11.8)$ \\
Other $(n, \%)$ & 33 \\
- Neurological disease & $(21.7)$ \\
- Cancer or hematologic malignancy discovery or & $10(6.7)$ \\
complications & $7(4.6)$ \\
- Inflammatory disease & $2(1.3)$ \\
- Cirrhosis complication & $3(2.0)$ \\
- Abdominal disease & $2(1.3)$ \\
- Locomotor disease & $2(1.3)$ \\
- Endocrinological disease & $1(0.7)$ \\
- Psychiatric disease & $2(1.3)$ \\
- Urological disease & $1(0.7)$ \\
- Social & $3(2.0)$ \\
\hline &
\end{tabular}

\section{Conclusion}

Since the patients who turned out to be COVID negative were initially suspected on the same basis as those who turned out to be positive, the differences observed highlight the relative utility of some characteristics in helping to distinguish genuine COVID-19 cases from other diseases. Since it can be advocated that rapid diagnosis (or diagnosis exclusion) of COVID-19 enables a better orientation of patients and avoids missed opportunities for uninfected patients, keeping in mind that these differences may help to better manage patients when/where very rapid diagnostic procedures are not yet available yet or not available all the time. This study also highlights the need for systematic heart function evaluation, as well as systematically looking for infections other than COVID-19. Last, it once again raises the question of the potential role of tobacco smoking in preventing COVID-19, which may still have to be further explored.
Acknowledgements We are thankful to all the doctors and interns of the Infectious Diseases Department at Dijon's teaching hospital for the help they gave us and all the work they accomplished during the epidemic. We thank Denise Mirat for editing assistance.

Author contribution Conceptualization, T.R., I.E. and L.P.; methodology, L.P.; formal analysis, T.R. and I.E.; investigation, T.R., I.E., F.M., T.S., M.A., L.B., A.C., L.M., S.M., M.B., A.S-R., P.C., M.D., F-X.C., C.E., Q.B., B.N., L.B., B.B., M.Bl. and L.P.; data curation, T.R. and I.E.; writing - original draft preparation, T.R., I.E., M.B. and L.P.; writingreview \& editing, T.R., I.E., M.Bl, L.P.; supervision, M.Bl. and L.P.

Data availability Anonymous data could be shared on demand.

Code availability Not applicable.

\section{Declarations}

Ethics approval Not applicable.

Consent to participate Not applicable.

Consent for publication Not applicable.

Conflict of interest The authors declare that they have no conflict of interest.

\section{References}

1. Zhu N, Zhang D, Wang W et al (2020) A Novel Coronavirus from Patients with Pneumonia in China, 2019. N Engl J Med 382(8): 727-733. https://doi.org/10.1056/NEJMoa2001017

2. Guan W-J, Ni Z-Y, Hu Y et al (2020) Clinical Characteristics of Coronavirus Disease 2019 in China. N Engl J Med 382(18):17081720. https://doi.org/10.1056/NEJMoa2002032

3. Wang D, Hu B, Hu C et al (2020) Clinical Characteristics of 138 Hospitalized Patients With 2019 Novel Coronavirus-Infected Pneumonia in Wuhan, China. JAMA 323(11):1061. https://doi. org/10.1001/jama.2020.1585

4. Wu C, Chen X, Cai Y et al (2020) Risk Factors Associated With Acute Respiratory Distress Syndrome and Death in Patients With Coronavirus Disease 2019 Pneumonia in Wuhan, China. JAMA Intern Med 13:934-943. https://doi.org/10.1001/jamainternmed. 2020.0994

5. Plaçais L, Richier Q (2020) COVID-19 : caractéristiques cliniques, biologiques et radiologiques chez l'adulte, la femme enceinte et l'enfant. Une mise au point au cœur de la pandémie. Re Méd Interne 41(5):308-318. https://doi.org/10.1016/j.revmed.2020.04. 004

6. COVID-19 : EN DIRECT. SFR e-Bulletin. Accessed Jul 21, 2020. https://ebulletin.radiologie.fr/covid19

7. Shi S, Qin M, Shen B et al (2020) Association of Cardiac Injury With Mortality in Hospitalized Patients With COVID-19 in Wuhan, China. JAMA Cardiol 25:802-810. https://doi.org/10.1001/ jamacardio.2020.0950

8. Vrsalovic M, Vrsalovic PA (2020) Cardiac troponins predict mortality in patients with COVID-19: A meta-analysis of adjusted risk estimates. J Infect 19:e99-e100. https://doi.org/10.1016/j.jinf.2020. 05.022

9. Pranata R, Huang I, Lukito AA, Raharjo SB (2020) Elevated Nterminal pro-brain natriuretic peptide is associated with increased 
mortality in patients with COVID-19: systematic review and metaanalysis. Postgrad Med J 96(1137):387-391. https://doi.org/10. 1136/postgradmedj-2020-137884

10. Xie S, Lei Z, Chen X et al (2020) Chest CT-based differential diagnosis of 28 patients with suspected corona virus disease 2019 (COVID-19). Br J Radiol 93(1112):20200243. https://doi.org/10. 1259/bjr.20200243

11. He J-L, Luo L, Luo Z-D et al (2020) Diagnostic performance between CT and initial real-time RT-PCR for clinically suspected 2019 coronavirus disease (COVID-19) patients outside Wuhan, China. Respir Med 168:105980. https://doi.org/10.1016/j.rmed. 2020.105980

12. Pasquereau A, Andler R, Arwidson P, Guignard R, Nguyen-Thanh V (2020) CONSOMMATION DE TABAC PARMI LES ADULTES : BILAN DE CINQ ANNÉES DE PROGRAMME NATIONAL CONTRE LE TABAGISME, 2014-2019. BEH 14: 273-281

13. Leung JM, Sin DD (2020) Smoking, ACE-2, and COVID-19: Ongoing Controversies. Eur Respir J 56(1):2001759. https://doi. org/10.1183/13993003.01759-2020

14. Leung JM, Yang CX, Tam A et al (2020) ACE-2 expression in the small airway epithelia of smokers and COPD patients: implications for COVID-19. Eur Respir J 55(5):2000688. https://doi.org/10. 1183/13993003.00688-2020

15. Smith JC, Sausville EL, Girish V, Yuan ML, John KM, Sheltzer JM (2020) Cigarette Smoke Exposure and Inflammatory Signaling
Increase the Expression of the SARS-CoV-2 Receptor ACE2 in the Respiratory Tract. Cell Biology 53:514-529.e3. https://doi. org/10.1101/2020.03.28.013672

16. Sanchis-Gomar F, Lavie CJ, Perez-Quilis C, Henry BM, Lippi G (2019) Angiotensin-Converting Enzyme 2 and Antihypertensives (Angiotensin Receptor Blockers and Angiotensin-Converting Enzyme Inhibitors) in Coronavirus Disease. Mayo Clin Pro 2020: S0025619620303153. https://doi.org/10.1016/j.mayocp.2020.03. 026

17. Lippi G, Sanchis-Gomar F, Henry BM (2020) Active smoking and COVID-19: a double-edged sword. Eur J Intern Med 77:123-124. https://doi.org/10.1016/j.ejim.2020.04.060

18. Lippi G, Henry BM (2020) Active smoking is not associated with severity of coronavirus disease 2019 (COVID-19). Eur J Intern Med 75:107-108. https://doi.org/10.1016/j.ejim.2020.03.014

19. Zhao Q, Meng M, Kumar R et al (2020) The impact of COPD and smoking history on the severity of COVID-19: A systemic review and meta-analysis. J Med Virol 92:1915-1921. https://doi.org/10. 1002/jmv. 25889

20. Menni C, Valdes AM, Freidin MB et al (2020) Real-time tracking of self-reported symptoms to predict potential COVID-19. Nat Med 26(7):1037-1040. https://doi.org/10.1038/s41591-020-0916-2

Publisher's note Springer Nature remains neutral with regard to jurisdictional claims in published maps and institutional affiliations. 